UCRL-ID-123847

\title{
Detection of Illegal Drugs Using Passive Infrared Sensing
}

C. L. Bennett

M. R. Carter

D. J. Fields
RECEIVED

AUG 121996

OSTI

\section{April 12, 1996}

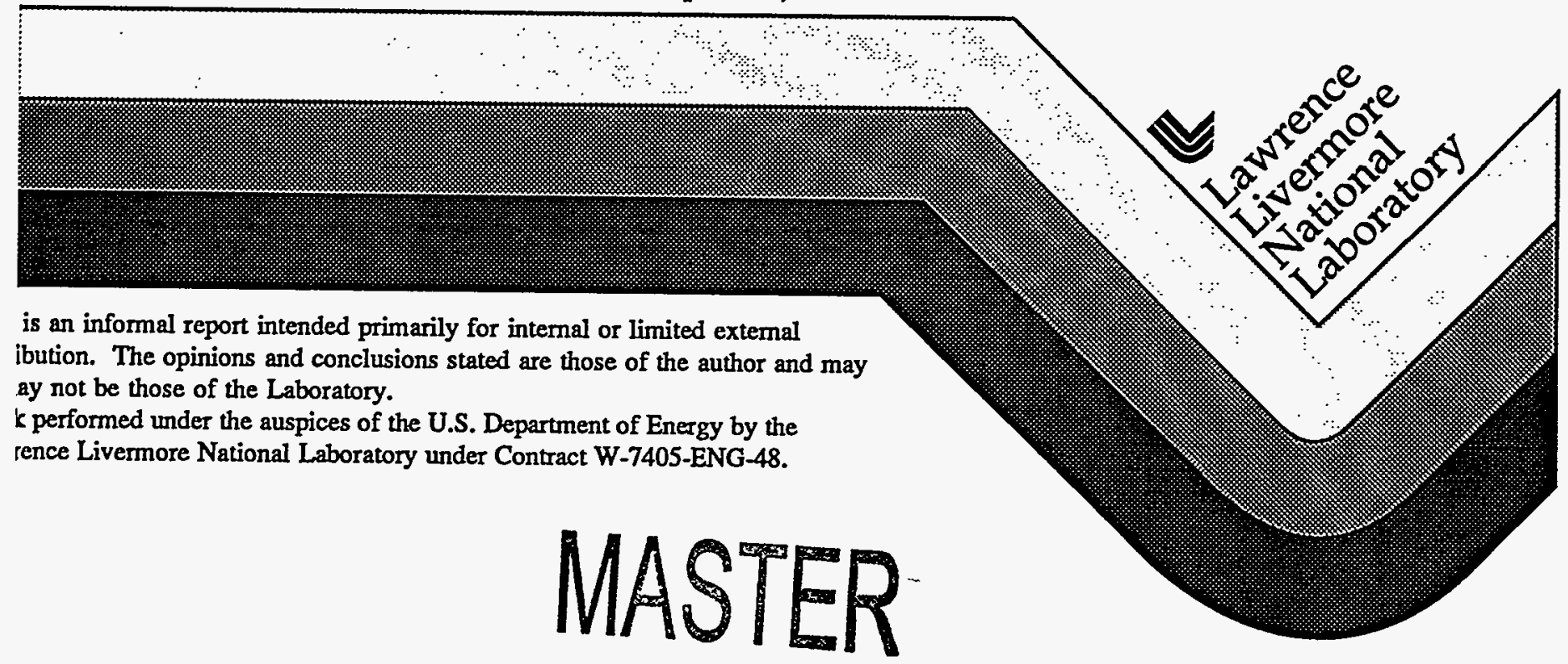


This document was prepared as an account of work sponsored by an agency of the United States Government. Neither the United States Government nor the University of California nor any of their employees, makes any warranty, express or implied, or assumes any legal liability or responsibility for the accuracy, completeness, or usefulness of any information, apparatus, product, or process disclosed, or represents that its use would not infringe privately owned rights. Reference herein to any specific commercial product, process, or service by trade name, trademark, manufacturer, or otherwise, does not necessarily constitute or imply its endorsement, recommendation, or favoring by the United States Government or the University of California. The views and opirions of authors expressed herein do not necessarily state or reflect those of the United States Government or the University of California, and shail not be used for advertising or product endorsement purposes.

This report has been reproduced directly from the best available copy.

Available to DOE and DOE contractors from the Office of Scientific and Technical Information

P.O. Box 62, Oak Ridge, TN 37831

Prices available from (615) 576-8401, FTS 626-8401

Available to the public from the National Technical Information Service

U.S. Department of Commerce 5285 Port Royal Rd. Springfield, VA 22161 


\section{DISCLAIMER}

This report was prepared as an account of work sponsored by an agency of the United States Government. Neither the United States Government nor any agency thereof, nor any of their employees, makes any warranty, express or implied, or assumes any legal liability or responsibility for the accuracy, completeness, or usefulness of any information, apparatus, product, or process disclosed, or represents that its use would not infringe privately owned rights. Reference herein to any specific commercial product, process, or service by trade name, trademark, manufacturer, or otherwise does not necessarily constitute or imply its endorsement, recommendation, or favoring by the United States Government or any agency thereof. The views and opinions of authors expressed herein do not necessarily state or reflect those of the United States Government or any agency thereof. 


\section{DISCLAIMER}

Portions of this document may be illegible in electronic image products. Images are produced from the best available original document. 


\title{
Detection of Illegal Drugs Using Passive Infrared Sensing
}

\author{
Charles L. Bennett, Michael R. Carter and David J. Fields \\ Lawrence Livermore National Laboratory
}

This report summarizes results from ARPA sponsored field experiments testing the feasibility of detecting illegal drugs using passive infrared spectroscopy in the 8-13 $\mu \mathrm{m}$ spectral band. We also discuss the basic concept being tested, summarize the various experiments performed and their results, and present a general conclusion from the experiments.

\subsection{Detection Concept}

The detection of drug contraband is an important but difficult problem. Those engaging in the smuggling of contraband attempt to operate as covertly as possible, typically concealing contraband in layers of wrapping and multiple containers. Even with all of these measures, trained "drug dogs" are remarkably successful in the detection of smuggled contraband. Evidently, residual fugitive vapors are present at some level which the dogs sense of smell is keen enough to detect. Even though a dog's abilities may be extremely good, it would be desirable to have affordable, practical instrumentation which could supply similar or complementary capabilities.

\subsection{Passive infrared spectroscopy}

Infrared spectroscopy has been used for many years for the detection and identification of chemical substances. The thermal infrared portion of the spectrum is particularly useful for remote chemical analysis, since almost all molecules have characteristic rotationvibration spectra in the infrared, and a broad portion of the so-called "fingerprint" region of the infrared spectrum lies where the atmosphere is relatively transparent, between 8 and $13 \mu \mathrm{m}$.

\subsection{Geometry}

Gaseous emissions in the line of sight between a source of infrared light (the backlighter) and an infrared spectrometer cause a loss of transmission at certain wavelengths. The characteristic absorption spectrum of a given sample may be used to identify and quantify the chemical compounds in the plume. This geometry is depicted in Figure 1. In addition to absorbing infrared radiation, the plume may emit in the infrared as well. In the case that the background is warmer than the gas plume, the spectrometer will measure an absorption spectrum as discussed above. When the gas is warmer 
than the background, an emission spectra will be measured. In the case where the gas temperature and background temperature are the same, the presence of the gas in undetectable at any concentrations.

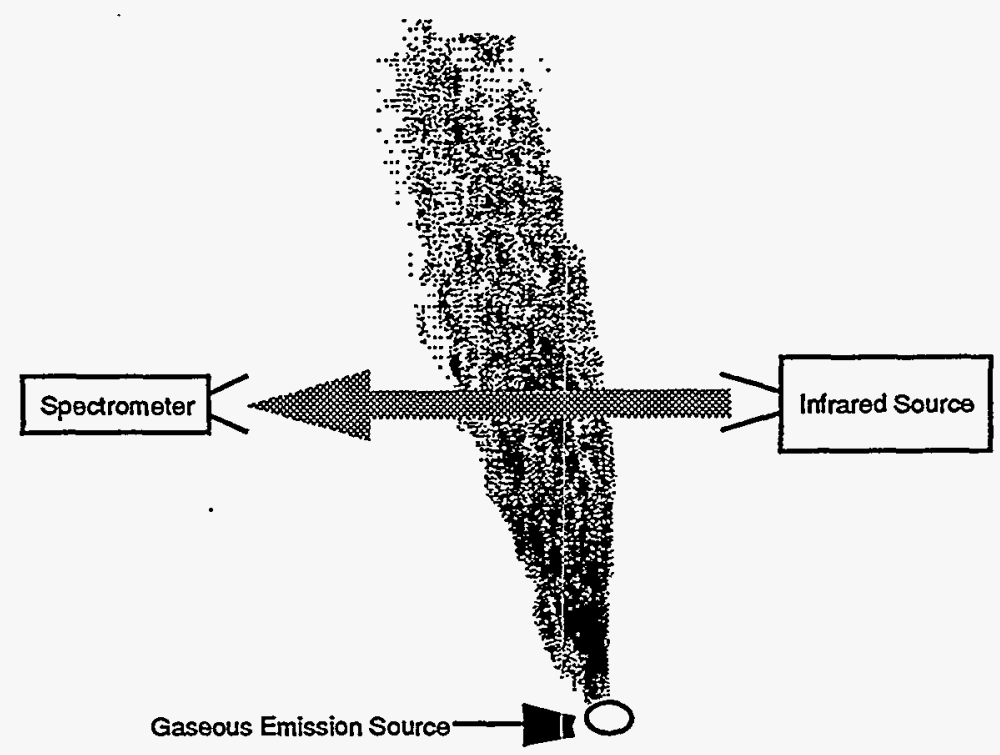

\section{FIGURE 1.}

The geometrical layout of a representative experiment is sketched. Experiments were performed with either an active hot source (a Nernst glower) or with a passive ambient temperature infrared source.

\subsubsection{Sources}

In some cases the source of infrared light may simply be the natural thermal emission of the background materials. In other cases the thermal light source is an artificial background, such as a hot plate or Nernst glower. The use of an artificial source provides a reproducible, high temperature source signal. Because these sources can be made quite hot, they also provide larger temperature contrasts with the plume, and thus larger potential signals.

\subsubsection{Laboratory vs. field geometries}

The geometry sketched in Figure 1 is commonly used in the laboratory, in the field, in remote sensing, and even in astronomy. In a controlled laboratory environment, the "plume" sketched in Figure 1. could instead be contained within a clean gas cell, where the concentration and temperature of the material can be controlled in order to enable high quality spectroscopic measurements. In the uncontrolled environment characteristic of field measurements, both the average concentration of the gas present in the line of sight and the thickness of the plume vary with the local wind conditions. Furthermore, when uncontrolled backlighters are used, the variance in the spectral intensities of the source can also make the detection and quantification problem more difficult.

\subsubsection{Enclosed environments}

In the more controlled environment available indoors, the vagaries of the outdoor wind conditions may be eliminated. However, even in the absence of wind, the concentration 
level of a given gaseous compound emanating from a given source is strongly dependent on such effects as the motion of people walking around in the room, the temperature of the compound of interest, the vapor pressure of the compound, the area of exposed surface. These can all affect the rate of transpiration of the compounds of interest. The advantage of a closed environment for the measurement of gaseous emissions is that eventually, given sufficient source material, the partial pressure of the gaseous emission becomes equal to its equilibrium vapor pressure.

\subsection{Application}

The use of passive infrared sensing could be applied where direct visual and physical examination of contraband carriers is not possible or is not practical. Examples might include the inspection of luggage, the surveillance of vehicles at choke points and the general inspection of shipping containers in a warehouse setting. In such situations, currently, dogs can be used to search for illegal drugs. This procedure is both expensive and time consuming. In addition, the attention span of dogs is limited. The use of an automatic system to cue dogs to suspect areas or containers would improve the utilization of the animals.

\subsection{Overview of Experiments}

Although laboratory analytical techniques allow the detection and characterization of minuscule amounts of material, it is not clear whether the detection thresholds obtainable in realistic field environments are anywhere near those required in a realistic contraband detection scenario. A series of experiments were conducted to explore this issue.

\subsection{Instrumentation}

Two instruments, both operating in the long wave Infrared region of the spectrum (8-12 $\mu \mathrm{m})$ were used to acquire the data for these experiments. Most of the data was acquired with a commercial FTIR spectrometer, manufactured by MIDAC, which is capable of high signal to noise ratio ( $S N R>1000$ ) measurements at high spectral resolution (up to $0.5 \mathrm{~cm}^{-1}$ ) in reasonably short times. Some of the data was acquired with the LIFTIRS (Livermore Imaging FTIR Spectrometer) instrument, which is an imaging FTIR spectrometer also capable of high spectral resolution (4. $\mathrm{cm}^{-1}$ ) and good SNR (SNR>200), but with the additional feature of forming complete hyperspectral images, where the spectrum of each pixel of a given scene is obtained.

\subsection{Summary of experiments}

The set of experiments described here and summarized in Table 1. was conducted in order to explore the impact of the various above mentioned factors on the detectability of drugs. The primary focus of the present study was cocaine, but some samples of marijuana were also examined. As a secondary compound, methyl benzoate was also studied, since it is formed when cocaine is exposed to the atmosphere. Methyl benzoate is of 
particular interest, since it is believed that it is this compound which is smelled by drug dogs.

The early outdoor methyl benzoate and drug simulant experiments were conducted at the Nevada Test Site during the June-July,1995 Desert Rainbow collection supported by ARPA/Tactical Technologies Office (TTO) Hyperspectral Mine Detection Program. Actual samples of illegal drugs were available for observation at the Thunder Mountain Evaluation Facility (TMEC) at Ft. Huachuka, Sierra Vista, Arizona. Both indoor and outdoor measurements of drugs and methyl benzoate were made at this facility. These experiments were conducted in September, 1995 in conjunction with the Thunder Rainbow collection supported by ARPA/TTO Hyperspectral Mine Detection Program.

TABLE 1.

Table 1. summarizes the measurements made. If no backlighter is specified, the existing background was used. DR and TR refer to Desert and Thunder Rainbow, respectively.

\begin{tabular}{|c|c|c|c|c|}
\hline Target substance & Location & Sensor & Backlighter & Result \\
\hline methyl benzoate & DR-Indoor & MIDAC & Nernst glower & Positive \\
\hline marijuana & DR-Indoor & MIDAC & Nernst glower & - \\
\hline cocaine & DR-Indoor & MIDAC & Nernst.glower & \\
\hline control & DR-Indoor & MIDAC & Nernst glower & \\
\hline methyl benzoate & DR-Outdoor & MIDAC & Nernst glower & Positive \\
\hline methyl benzoate & DR-Outdoor & MIDAC & ground & \\
\hline methyl benzoate & DR-Outdoor & MIDAC & wood & Positive \\
\hline methyl benzoate & DR-Outdoor & MIDAC & metal plate & Positive \\
\hline cocaine & TR-Outdoor & MIDAC & Nernst glower & \\
\hline cocaine & TR-Outdoor & MIDAC & ground & \\
\hline cocaine & TR-Outdoor & MIDAC & wood & \\
\hline cocaine & TR-Outdoor & MIDAC & metal plate & \\
\hline cocaine & TR-Car trunk & MIDAC & sky & \\
\hline cocaine, powder on paper & TR-Outdoor & MIDAC & reflected sky & \\
\hline cocaine, traces of powder on paper & TR-Outdoor & MDAC & reflected sky & \\
\hline paper alone, control & TR-Outdoor & MIDAC & reflected sky & \\
\hline cocaine block & TR-Outdoor & MIDAC & reflected sky & Positive \\
\hline plastic tape & TR-Outdoor & MIDAC & reflected sky & \\
\hline cocaine simulant & TR-Outdoor & MIDAC & ground & \\
\hline high explosive simulant & TR-Outdoor & MIDAC & ground & \\
\hline marijuana simulant & TR-Outdoor & MDAC & ground & \\
\hline cocaine & TR-Outdoor & LIFTIRS & wood & \\
\hline marijuana & TR-Outdoor & LIFTIRS & wood & \\
\hline methyl benzoate & TR-Outdoor & LIFTIRS & wood & \\
\hline
\end{tabular}




\subsection{Methyl benzoate experiments}

Methyl benzoate is formed when cocaine is exposed to the air, as it reacts with water. It is probably the chemical most easily sensed by dogs. Because of this fact and the availability of the compound in deciliter quantities, the initial experiments were performed with methyl benzoate as the target compound. The chemical properties of methyl benzoate are listed in Appendix 1.

\subsection{Indoor experiments}

Cocaine held in storage in indoor areas might be expected to emit methyl benzoate. Depending on the level of ventilation, this may produce concentrations detectable to $\mathbb{R}$ spectroscopy.

\subsubsection{Description of experiment}

An arrangement similar to that sketched in Figure 1 was used for observations of the vapors from a methyl benzoate sample. Sufficient methyl benzoate was poured into a 9" diameter container to cover the bottom with liquid. The container was then covered and placed outside to sit in the sun for a period of at least one hour. The unopened container was then placed approximately 3 inches under the line of sight between the spectrometer and a Nemst glower. The distance from the spectrometer to the light source was approximately 2 feet. A series of spectra were taken at 10 second intervals, with each spectrum being the sum of four scans, each taken at $0.5 \mathrm{~cm}^{-1}$ spectral resolution, integrating for approximately 6 seconds. Changes in conditions were made in the 4 free seconds between each spectra The various conditions are summarized in Table 2.

TABLE 2.

Summary of conditions for indoor methyl benzoate experiments.

\begin{tabular}{|l|l|l|}
\hline Spectrum \# & Status of container & Backlighter \\
\hline $1-2$ & closed & Nernst glower \\
\hline 3 & not present & ambient blackbody \\
\hline $4-7$ & closed & Nernst glower \\
\hline $8-12$ & open & Nernst glower \\
\hline 13 & not present & ambient blackbody \\
\hline $14-15$ & open & Nernst glower \\
\hline $16-19$ & removed & Nernst glower \\
\hline 20 & not present & ambient blackbody \\
\hline $21-29$ & removed & Nernst glower \\
\hline 30 & not present & ambient blackbody \\
\hline
\end{tabular}




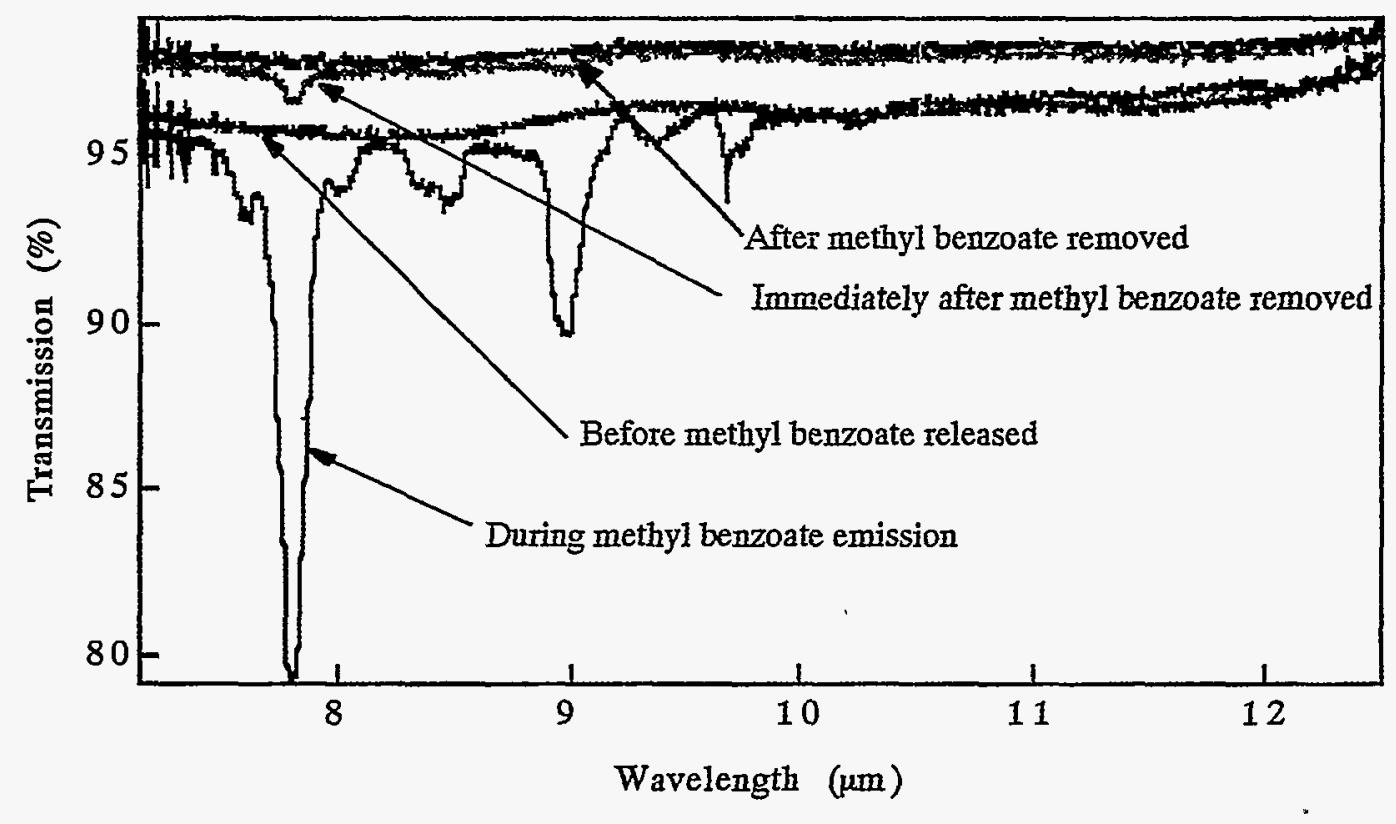

FIGURE 2.

Four spectra from the methyl benzoate experimental series are shown. The spectra from highest to lowest average transmission correspond to methyl benzoate removed (\#29), immediately after methyl benzoate removed $(\# 16)$, before methyl benzoate release $(\# 8)$, and during release (\#7). Note that the absorption peak is rapidly reduced as the vapors dissipate after the container is removed.

The ambient blackbody spectra was used to define the room background, and was subtracted from all other spectra. A spectrum taken 15 minutes prior to the initial emplacement of the container was used to define the $100 \%$ transmission level. All the background subtracted spectra were normalized to this reference spectrum, thus yielding absolutely calibrated transmission spectra.

The resulting transmission spectra allow accurate quantification of the level of the vapors present in the line of sight between the spectrometer and the backlighting infrared source. .

Sample spectra from the complete series of measurements are shown superimposed in Figure 2. The large dips in the spectrum taken during the release are attributed to the presence of methyl benzoate in the line of sight between the infrared source and the detector. The peak absorbance is approximately $20 \%$ at $7.8 \mu \mathrm{m}$.

During the experiment a small change in the overall level of the transmission seen before and after the methyl benzoate release is attributed to a slight baseline shift, as may result from a slight change in the alignment of the hot backlighter. This shift is typical and is not regarded as significant. 


\subsubsection{Quantiflcatlon of Indoor methyl benzoate experiments}

The absorbance spectrum calculated from the spectral transmittance (\#7) is compared in Figure 3 with the absorbance spectrum for pure methyl benzoate from the Sadtler reference library. The quality of agreement between the library reference and the field data spectrum is very good, and demonstrates that the confidence in the identification of the spectral features observed in the indoor field experiment with pure vapor phase methyl benzoate is very high.

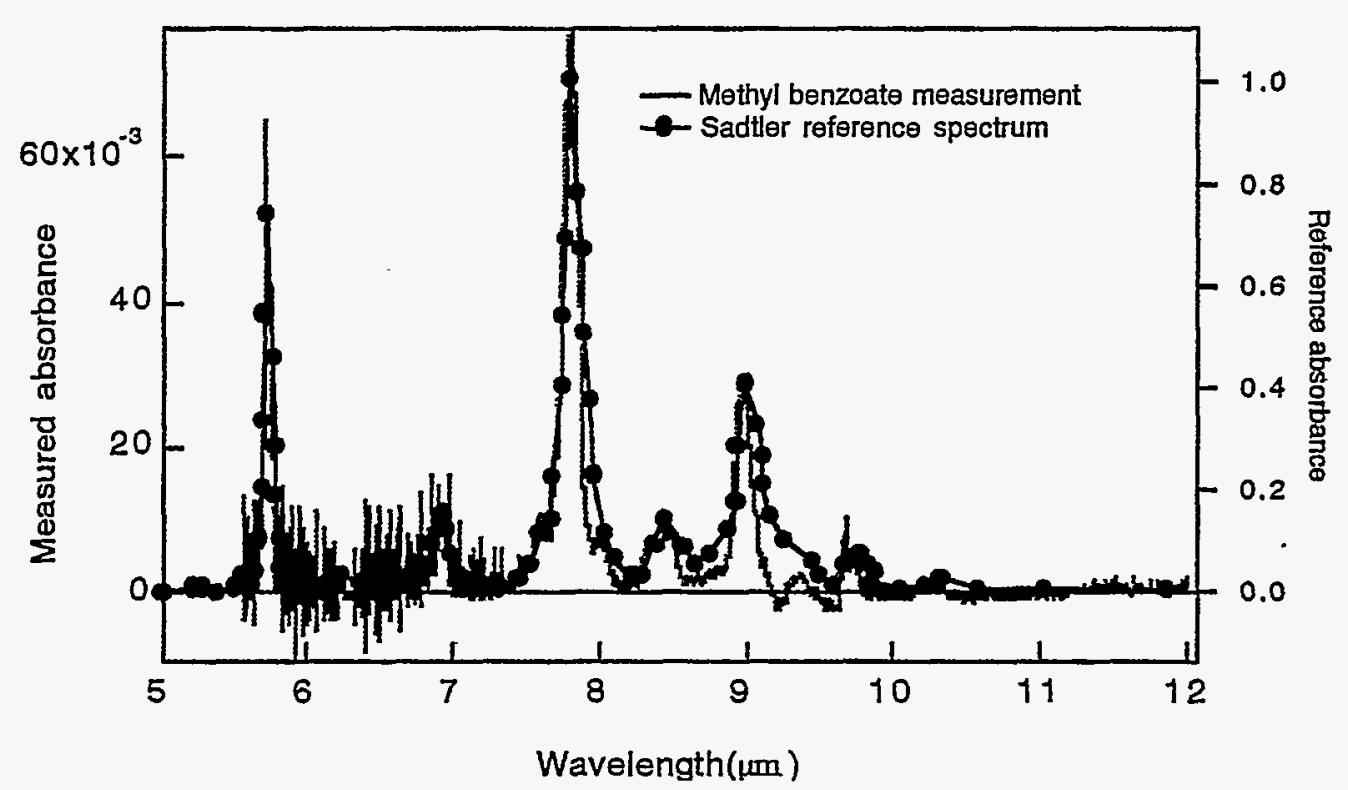

FIGURE 3.
The spectrum observed from evaporating methyl benzoate is compared with the Sadtler reference library spectrum for vapor phase methyl benzoate. The library reference spectrum corresponds to a spectral resolution of $4 \mathrm{~cm}^{-1}$ while the measured data were taken at a spectral resolution of $0.5 \mathrm{~cm}^{-1}$

In view of the agreement between the field spectrum and the library reference spectrum, it is possible to accurately quantify the integrated concentration length product for the methyl benzoate vapors observed in the field. For spectrum number 8 shown in Figure 2 , the transmission of the methyl benzoate corresponds to a concentration length product of $176 \mathrm{ppmm}$. Averaged over the 2 foot path length, the path averaged concentration of the methyl benzoate is $289 \mathrm{ppm}$, or 0.2 Torr partial pressure, which is approximately $50 \%$ of the methyl benzoate vapor pressure at standard temperature, and roughly $20 \%$ of the vapor pressure of the material under the desert heat conditions.

\subsubsection{Dissipation of vapor In Indoor environment}

After the container holding the liquid methyl benzoate was removed from the trailer, the vapors were observed to quickly dissipate. In Figure 2, the spectrum taken immediately after the container was removed, exhibits a methyl benzoate peak absorbance of approximately $0.9 \%$, which is about an order of magnitude below the value during the release. 
Later, (\#17), the methyl benzoate peak absorption has decreased to approximately $0.2 \%$, which is only a factor of 2 above the detection threshold for a single spectral channel of roughly $0.1 \%$ absorption. This detection threshold level corresponds to a path averaged concentration of approximately $1.5 \mathrm{ppm}$, or a path integrated concentration level of 1 ppmm.

Using the integrated absorbance above background between the limits 7.5 to $8.1 \mu \mathrm{m}$, which corresponds to the region around the most intense peak in the methyl benzoate spectrum, the time variation of the integrated concentration length product for the vapors was measured as a function of time after the container was initially opened. This data is plotted in Figure 4. The detection threshold for this integrated absorbance is approximately $200 \mathrm{ppm}$. Thus, after approximately 2 minutes the average concentration has decreased below threshold.

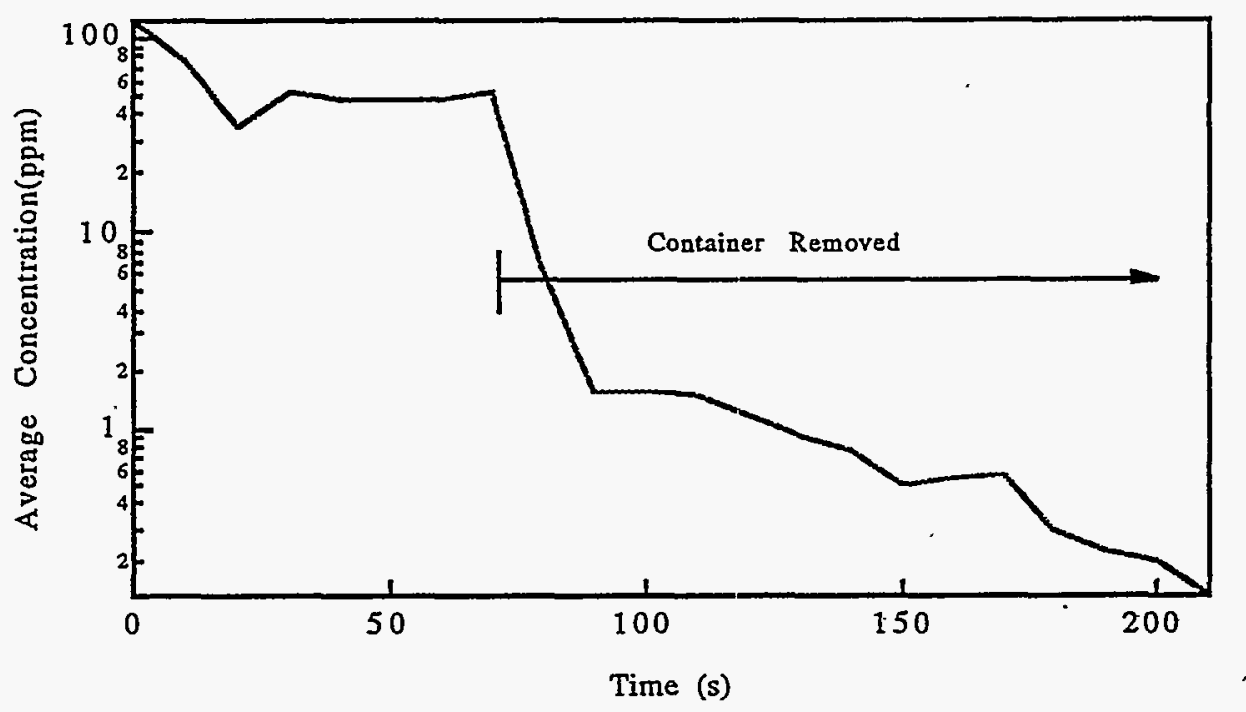

FIGURE 4.

The average concentration of methyl benzoate along the line of sight between the infrared source and detector is plotted as a function of time, starting from the initial opening of the container. The measured concentration drops rapidly after the source of methyl benzoate is removed.

\subsection{Outdoor methyl benzoate experiment}

A series of outdoor measurements were made with methyl benzoate liquid. The container was placed on a variety of substrates, including a road bed and a wooden plywood board, with these substrates acting as backlighters for the observation of the vapors. A line of sight from the infrared spectrometer to the background thus passed over the liquid at a height of approximately one foot.

In order to compensate for the expected loss of detectability in outdoor measurements, some spectral measurements were made at lower spectral resolution. These were made 
at a spectral resolution of $4 \mathrm{~cm}^{-1}$ with an approximate noise level of $0.01 \% \mathrm{rms}$. This should be compared to a noise level of $0.1 \% \mathrm{~ms}$ at a spectral resolution of $0.5 \mathrm{~cm}^{-1}$.

\subsubsection{Results for outdoor methyl benzoate experiment}

For the case with the highest concentration of gas observed in all of the outdoor experiments with natural background, the strongest absorption in the spectrum was only about $0.3 \%$, at approximately $1270 \mathrm{~cm}^{-1}$. Such a spectrum is shown in Figure 5. Thus, the maximum outdoor signal is two orders of magnitude weaker than the typical signal seen with the indoor experiment. The general conclusion which may safely be drawn from this observation is that a substantially higher likelihood of detecting gaseous emissions will be obtained with the indoor experiments and artificial backlighters.

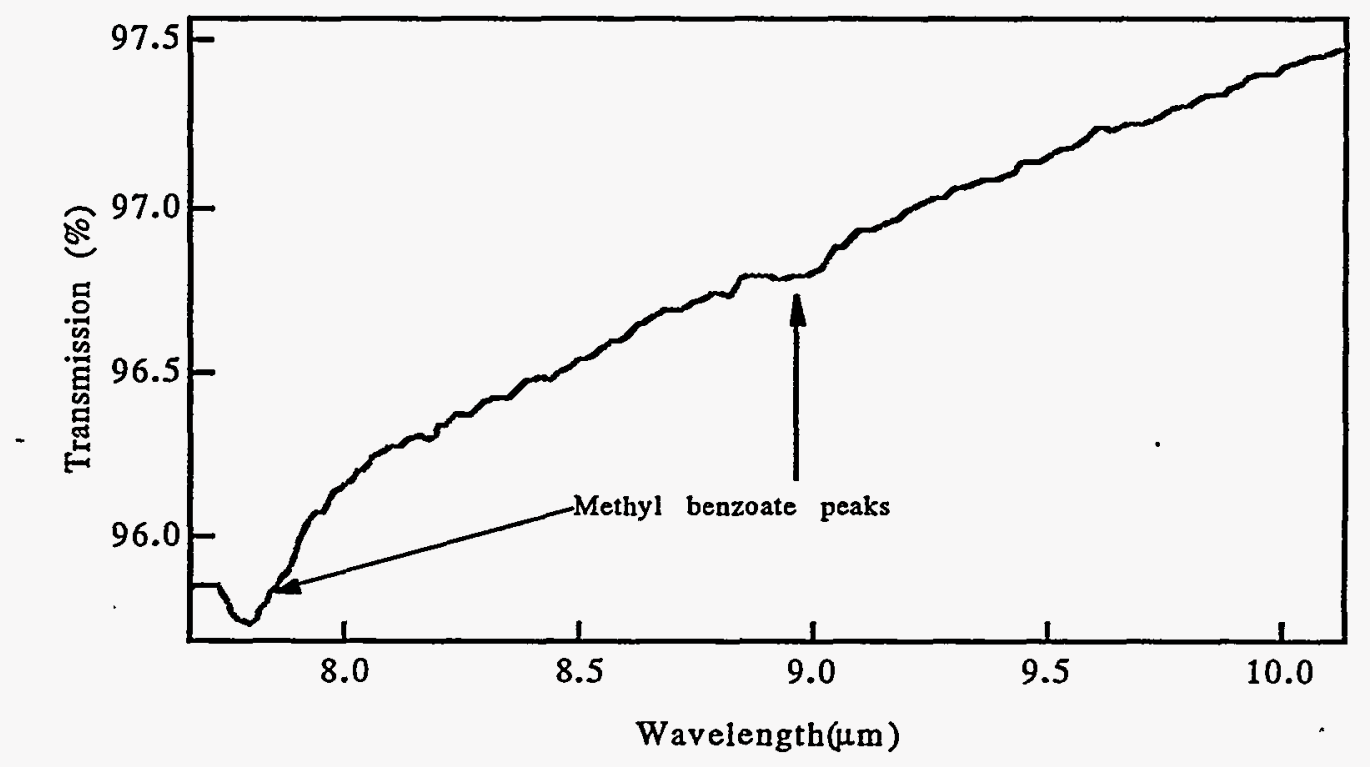

FIGURE 5.

Two methyl benzoate absorbance features are visible in the ratio of a spectrum with methyl benzoate present to a spectrum with only a wooden board in the background. The reason for the overall slope of this ratio may be attributed to a temperature difference of approximately $3^{\circ} \mathrm{C}$ between the backgrounds in the two measurements.

\subsubsection{Reasons for loss of apparent sensitivity in outdoor experiments}

The reason for this much higher apparent sensitivity with indoor experiments is threefold:

- First, with the indoor experiments, the gaseous vapors are not quickly dispersed and diluted by the wind. The available signature is therefore substantially larger.

- Second, the brightness temperature contrast between the hot backlighting filament and the temperature of the gases being evolved from a source in the indoor experiment is nearly $100 \%$, whereas in the outdoor case the contrast between the brightness temperature of the background and the ambient air temperature may be only a 
few degrees to perhaps 10 degrees, and thus less than a $10 \%$ radiance contrast. Both of these factors are highly variable, indeed they depend on the weather, and plausibly produce the loss of two orders of magnitude in sensitivity.

- Finally, the conditions of the indoor experiments are much more stable in time, and the variations of the background reference radiance levels, which are typical of outdoor measurements do not increase the clutter level for the indoor experiments.

\subsection{Indoor Drug Experiments}

\subsection{Indoor cocaine measurements}

A series of spectra was taken with a cocaine sample using the procedure above. The cocaine sample consisted of a brick of cocaine, approximately $2^{\prime \prime} \times 2 " \times 12$. This brick was contained in a foil and cardboard wrapping. A hole was made in the wrapping, and a surface area of approximately 1 square inch of bare cocaine was exposed. The entire brick was placed inside a closed container and was allowed to "cook" in the sun for approximately one hour before the measurements were made. When the measurements were made, the container with the cocaine was placed under the line of sight between the infrared source and detector. The detailed sequence of measurements for the cocaine case are itemized below in Table 3.

\section{TABLE 3.}

Summary of conditions for indoor cocaine experiments.

\begin{tabular}{|l|l|l|}
\hline Spectrum \# & Status of container & Backdighter \\
\hline 1 & removed & Nernst glower \\
\hline 2 & not present & ambient blackbody \\
\hline $3-4$ & removed & Nernst glower \\
\hline $6-9$ & open & Nernst glower \\
\hline $10-11$ & closed & Nernst glower \\
\hline 12 & not present & ambient blackbody \\
\hline $13-14$ & closed & Nernst glower \\
\hline $15-17$ & open & Nernst glower \\
\hline $18-25$ & removed & Nernst glower \\
\hline 26 & not present & ambient blackbody \\
\hline 27 & removed & Nernst glower \\
\hline
\end{tabular}

Examples of the spectra from these measurements are shown in Figure 6. There is no obvious sign of the methyl benzoate peak present in the data at the instrument sensitivity level of approximately at the $0.1 \%$ level, corresponding to a path averaged concentration of approximately $1.5 \mathrm{ppm}$. 


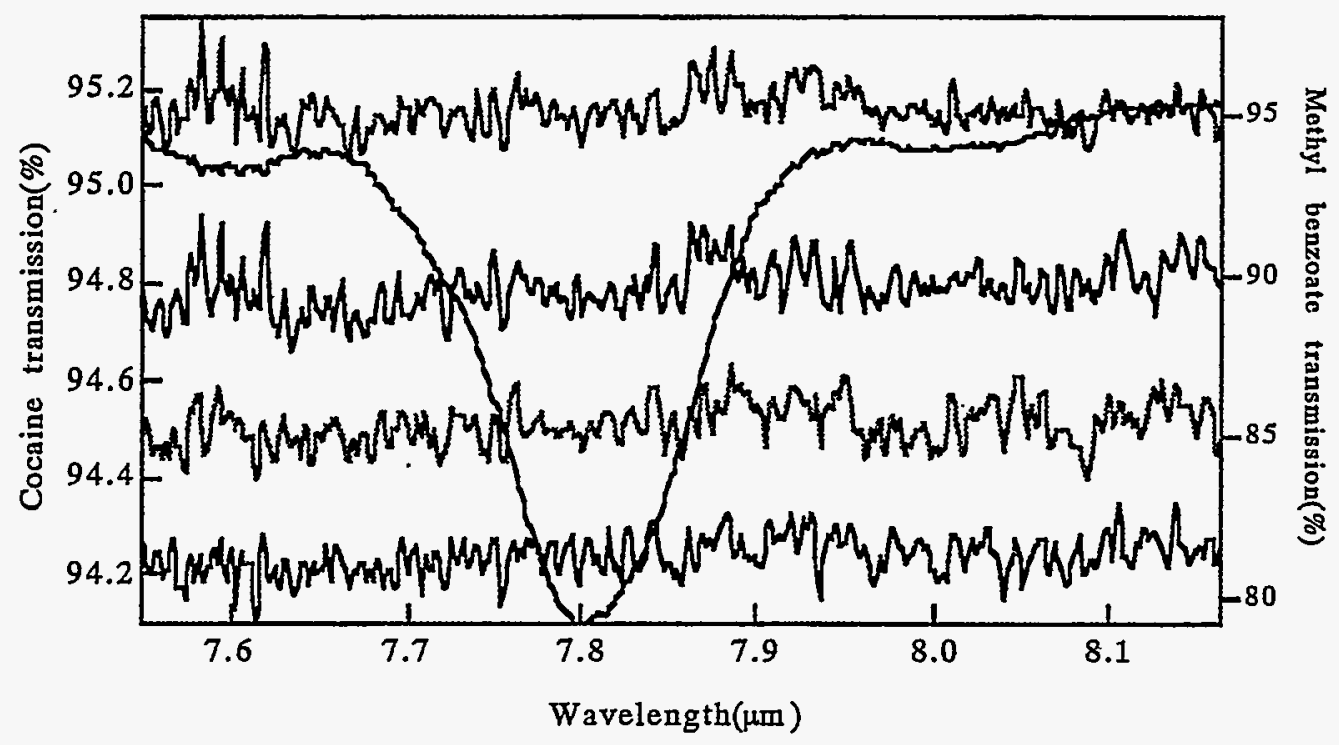

FIGURE 6.

TABLE 4.

Spectra measured of vapors above a cocaine sample in an indoor setting show no spectral features indicative of cocaine. The spectral range from 7.55 to $8.15 \mu \mathrm{m}$ is displayed for the averages of several sequences of spectra. (From the top, $\# 6-\# 9$, \#10$\# 11, \# 13-\# 14$, and $\# 15-\# 17)$. Each of these cases has been slightly offset vertically for clarity of display, with the earliest cases displayed highest on the plot. The scale on the left hand axis corresponds to the average of spectra 15-17. In addition, for reference, spectrum number 7 from Figure 2 is also displayed, according to the scale on the right hand axis, in order to show the position and shape of the peak expected for methyl benzoate.

\subsection{Indoor marl]uana experlments}

With exactly the same general procedure as described above in the above sections for methyl benzoate and cocaine $\mathrm{HCl}$, a series of spectra was taken with a marijuana sample. This sample consisted of a plastic bag containing relatively loosely packed marijuana. This bag was placed inside a closed container. When the measurements were made, the container with the marijuana was placed under the line of sight between the infrared source and detector.

Summary of conditions for indoor marijuana experiments.

\begin{tabular}{|l|l|l|}
\hline Spectrum \# & Status of contalner & Backlighter \\
\hline $1-3$ & closed & Nernst glower \\
\hline 4 & behind source & ambient blackbody \\
\hline $5-6$ & closed & Nernst glower \\
\hline $7-14$ & open & Nemst glower \\
\hline $15-17$ & removed & Nernst glower \\
\hline 18 & behind source & ambient blackbody \\
\hline $19-33$ & removed & Nemst glower \\
\hline
\end{tabular}


In Figure 7, the average of all spectra taken with the marijuana present is compared with the average of all spectra taken without the marijuana container present. The complete sequence of measurements for this case are itemized in Table 4.

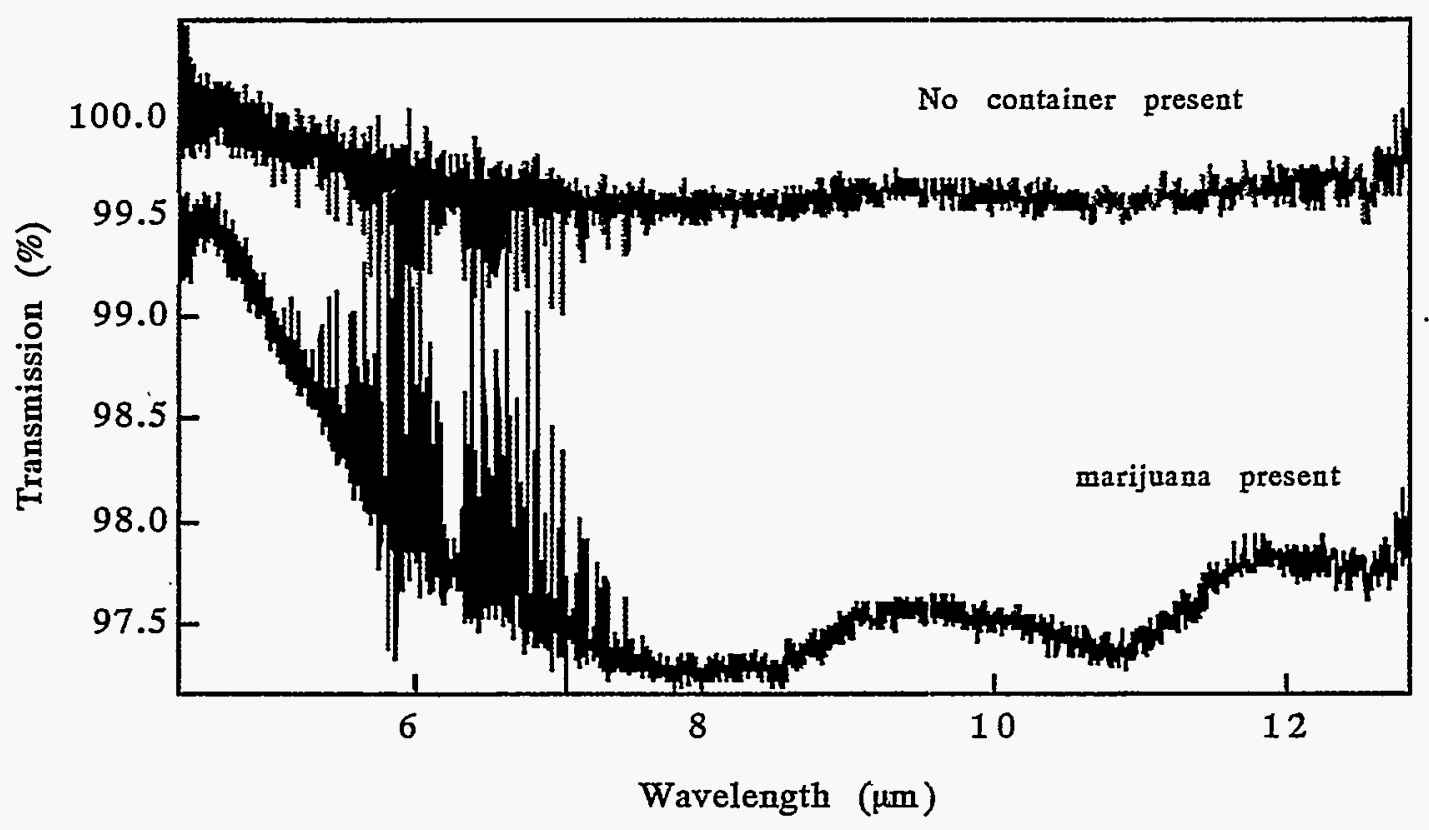

FIGURE 7.

Representative spectra observed with and without a container of marijuana present under the line of sight shows no indication of marijuana. The fine line structure between 5 and $7 \mu \mathrm{m}$ is due to water vapor. The general decrease in transmission for the case with the container present is attributed to a slight baseline drift and distortion related to positioning of the hot backlighting filament and is unrelated to marijuana.

\subsection{Other Non-imaging Outdoor Experiments}

\subsection{Synthetic drugs}

Aseries of measurements with synthetic Marijuana, synthetic high explosives, synthetic cocaine, and synthetic heroin were made as described above. These experiments were conducted at the Nevada Test Site in conjunction with the Desert Rainbow collection supported by ARPA/TTO Hyperspectral Mine Detection Program. 
In all of these experiments the approximate statistical noise level was $0.01 \% \mathrm{rms}$ absorption. In some cases systematic trends in the shape of the spectral background were at the level of $0.05 \%$, and correspondingly the threshold sensitivity was reduced. In no cases other than the liquid methyl benzoate was a significant signal seen outdoors.

\subsection{Drug measurements at TMEC}

A series of other outdoor experiments were also performed with the cooperation of the TMEC personnel and equipment. These experiments were conducted in conjunction with the Thunder Rainbow collection supported by ARPA/Operations Other-Than-War Hyperspectral Mine Detection Program.

\subsubsection{Wrapped cocaine against sky}

The cocaine was wrapped in plastic and aluminum foil, and placed within a cardboard box. The cardboard box was placed in a plastic bag, and stored within the trunk of an automobile. This elaborate containment was in fact the containment used by the drug smugglers at the time that the contraband was discovered by a drug dog. The particular sample used in this study was previously used evidence in a drug prosecution case, and was soon to be destroyed. The experiment conducted for this work consisted of setting up a line of sight directed towards a blue sky background. This produces a very high thermal contrast for most spectral features between approximately $8-13.3 \mu \mathrm{m}$. A series of spectra were acquired before, during, and after the trunk of the automobile containing the contraband was opened. Using the spectra before the trunk was opened as a baseline reference, the spectra observed after the trunk was opened were searched for significant spectral features. None were found.

\subsubsection{Partially unwrapped cocaine}

Cocaine, with all wrapping except the innermost removed was placed immediately under the path of an infrared beam, and vapors vented into the beam were searched for. No significant peaks were found.

\subsubsection{Wrapped cocaine against panel}

Cocaine in its wrapper was placed on a black panel background and vapors were searched for. Nothing significant was found.

\subsubsection{Vapors from exposed cocaine}

Cocaine, with approximately a 1 square inch patch of exposed surface was placed immediately under the path of an infrared beam, and vapors vented into the beam were searched for. Nothing significant was found.

\subsubsection{Cocaine residues on paper}

Visible cocaine powder residues were placed on a sheet of paper. The direct infrared spectra from these residues were searched for. The complex spectra observed were consistent with being clean paper.

\subsubsection{Direct observation of cocaine}

An exposed patch of the pure cocaine was viewed directly with the infrared spectrometer (with some portion of the surrounding wrapping material in the field of view of the instrument). A spectrum of the wrapping material itself was subtracted from the mixed spectrum of cocaine and wrapping paper. The resulting reflectance spectrum was attrib- 
uted to pure cocaine, and is displayed in Figure 8 . It is most significant that the reflectance spectrum of the cocaine is very dissimilar to the absorbance spectrum of the methyl benzoate vapors, which is supposed to be the dominant volatile compound evolved from cocaine upon exposure to air.

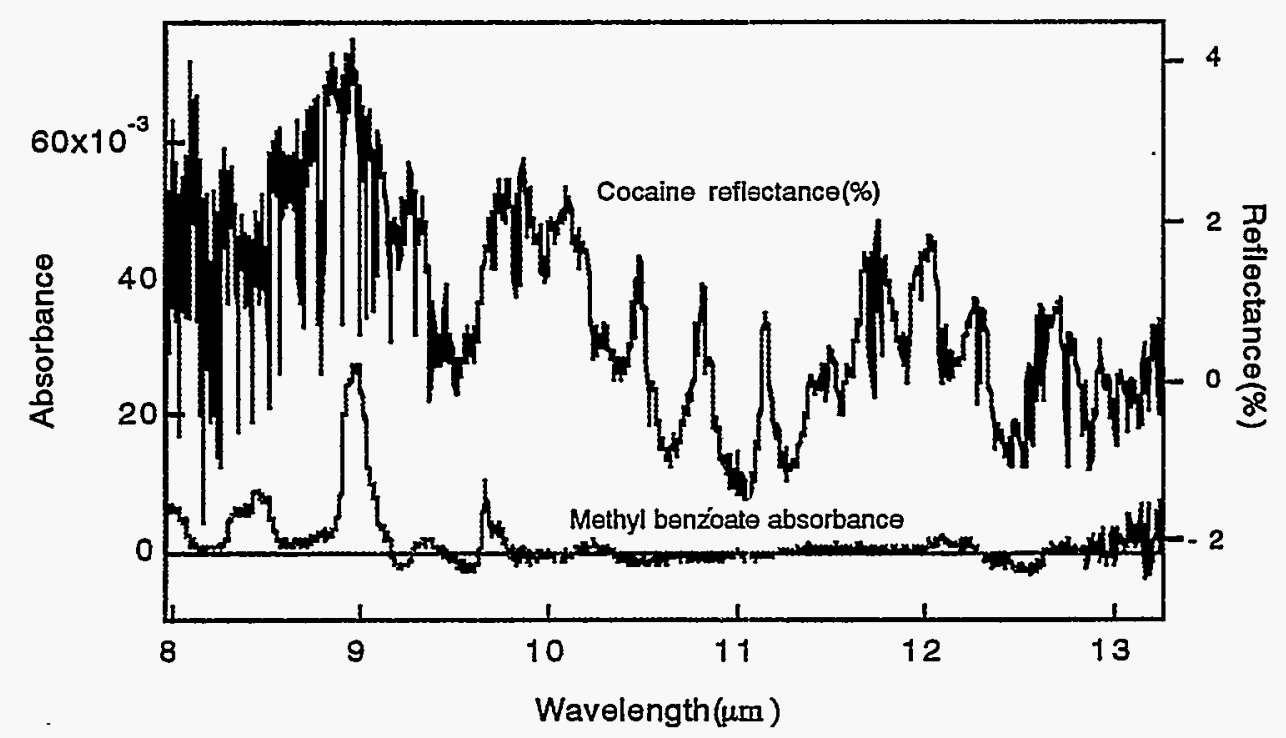

FIGURE 8.

\subsection{Imaging spectroscopic experiments}

In conjunction with the Thunder Rainbow experimental campaigns a series of imaging experiments were conducted with the LIFTIRS instrument concurrently with the nonimaging MIDAC measurements. These measurements were performed before it was clear that the outdoor signal to noise levels wrould almost certainly be too low to be able to detect any significant results with the LJFTIRS instrument. Indeed, no signals were seen from any of the samples observed, which included marijuana, cocaine, and methyl benzoate.

An example of the data from the LIFTIRS instrument is displayed in Figures 9 and 10 for the methyl benzoate experiment, which would be expected to have the largest signal of the three experiments. In Figure 9, an image is shown, which corresponds to a single slice of the hyperspectral data cube which is measured by the imaging spectrometer. The image displayed in Figure 9 corresponds to the scene immediately after the container of methyl benzoate was opened. Another data set was also acquired immediately before the container was opened. Any difference in the spectral radiance produced by vapors emanating from the container can thus be determined by subtracting the spectra seen before and after the container was opened. 
From the set of difference spectra corresponding to the locations indicated in Figure 9, which are displayed in Figure 10, it can be seen that no significant spectral difference is apparent. Indeed, from the estimated noise level in the spectra; and the previously observed signal levels from the outdoor experiments of approximately $0.3 \%$ at the most, it is clear that the present imaging spectroscopic data have insufficient SNR to be able to detect vapors with passive backlighting at even the highest expected concentration levels.

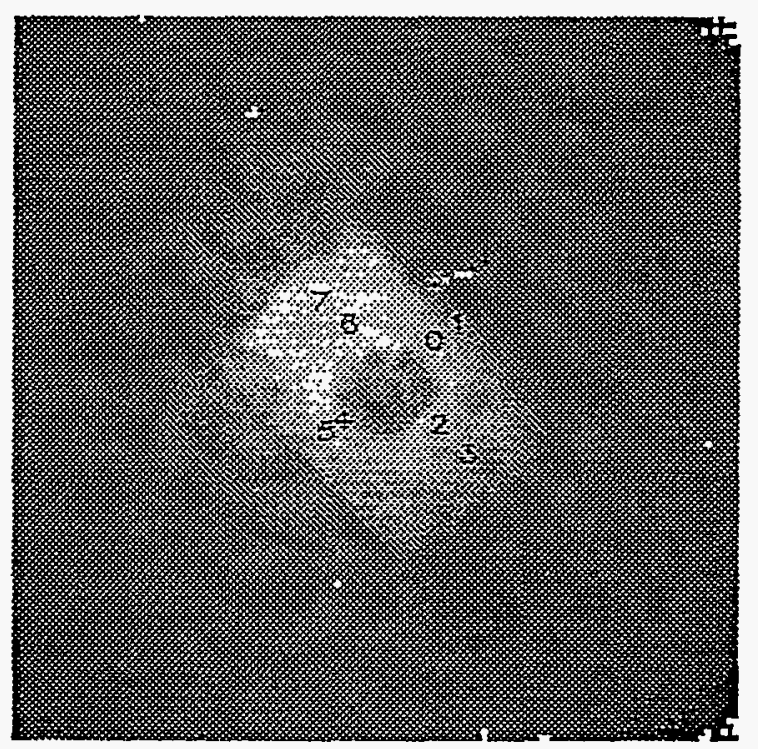

FIGURE 9.

An image from LIFTIRS measurements of a methyl benzoate sample, taken over a single spectral channel. The circular object near the center of the fields of view is a container holding a quantity of methyl benzoate liquid. Eight spatial sample points are indicated by number in this figure. At each of these eight sample points the difference in the emission spectra take before and after the container was opened have been calculated and displayed in Figure 10. 


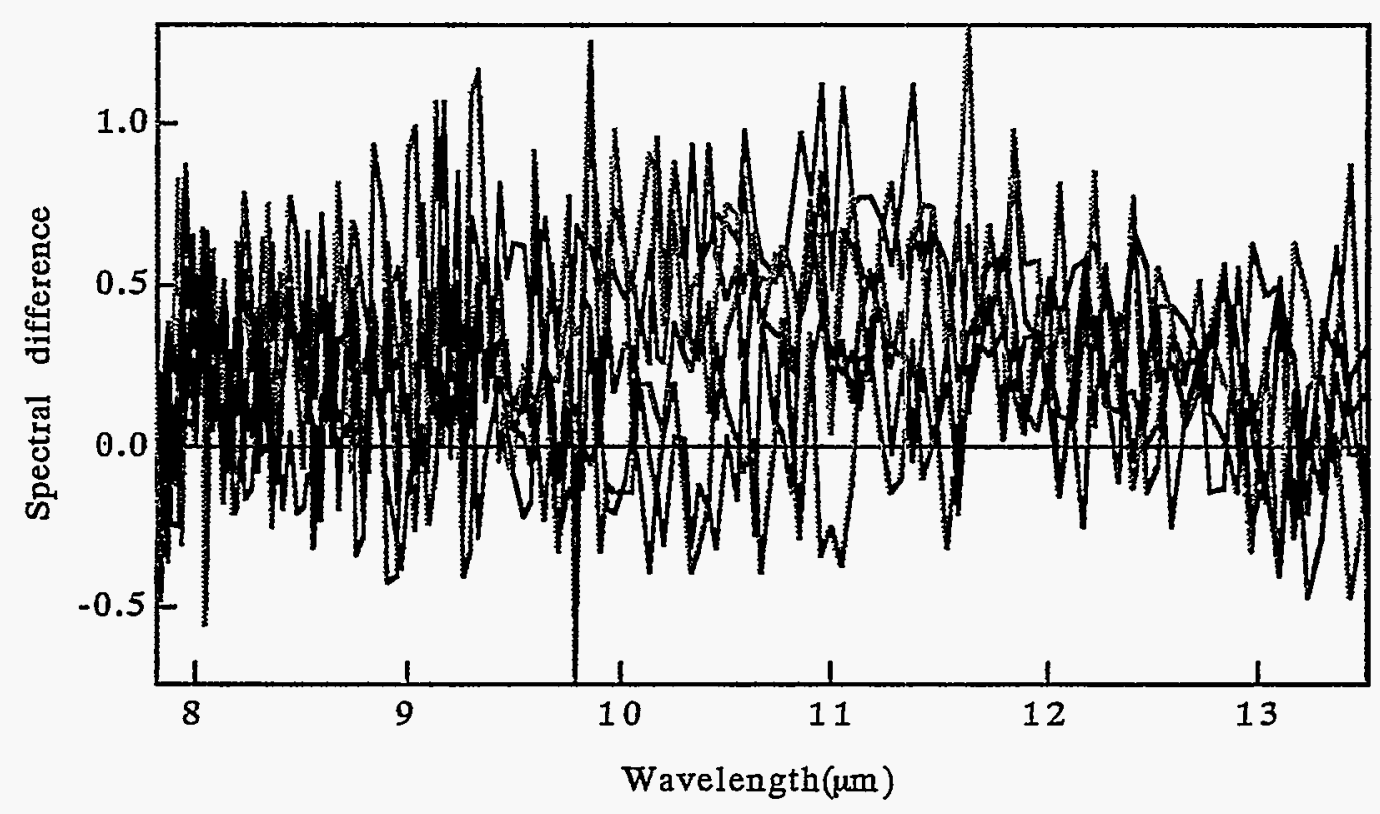

FIGURE 10.

Difference spectra corresponding to positions indicated in Figure 9 are displayed. No significant spectral features are seen at any wavelength. in particular, no significant evidence for the presence of methyl benzoate is seen in this data.

\subsection{CONCLUSIONS}

The feasibility of detecting concealed contraband drugs by infrared remote sensing of the vapors which emanate from them was studied in a number of controlled field measurements of both illicit materials and their surrogates. Measurements in a variety of quasi-realistic scenarios were studied. In none of these cases was any signal above background levels observed for any of the real or synthetic drug compounds. Only a very weak signal for vapors emanating from pure liquid methyl benzoate (which is a secondary reaction product of cocaine with the atmosphere) was observed in the outdoor, natural backlighting experiments. Even in the most favorable setting, real drug compounds were not detected. Thus we conclude that passive infrared spectroscopy is not practical for the direct detection of cocaine or marijuana contraband.

\subsection{ACKNOWLEDGMENTS}

This work was performed for ARPA under contractAO-C712, and under the auspices of the U.S. Department of Energy under Contract No. W-7405-Eng-48. We thank Kalon and Mark Genz of the Cochise County Sheriff's department for kindly supplying the drug samples used in this work. We also thank Stacy Wright of the Thunder Mountain Evaluation Center (TMEC) for allowing us to stage our experiments at the TMEC facility. We thank John Pennella and Trent DePersia of ARPA for sponsoring this work. We would like to thank Anu Bowman of Space Applications Corporation and Ed Winter of 
Technical Research Associates for their help in motivating and executing these experiments. We also thank the participants of the Desert Rainbow and Thunder Rainbow Hyperspectral Mine Detection Experimental program for providing important assistance in helping to set up and carry out some of the measurements made in this work.

\subsection{Appendix 1: Properties of methyl benzoate}

The properties of methyl benzoate are summarized in Table 5. The vapor pressure as a function of temperature is shown in Figure 11.

TABLE 5.

FIGURE 11.
Properties of methyl benzoate

$\begin{array}{ll}\text { Property } & \text { Value } \\ \text { Melting Point } & -12.4^{\circ} \mathrm{C} \\ \text { Boiling Point } & 199.5^{\circ} \mathrm{C} \\ \text { Density (@20 } & 1.0888 \\ \text { Vapor Pressure (STP, torr) } & 0.3944 \\ \text { Molecular Weight (daltons) } & 136.15\end{array}$

The vapor pressure for pure methyl benzoate is displayed as a function temperature. 


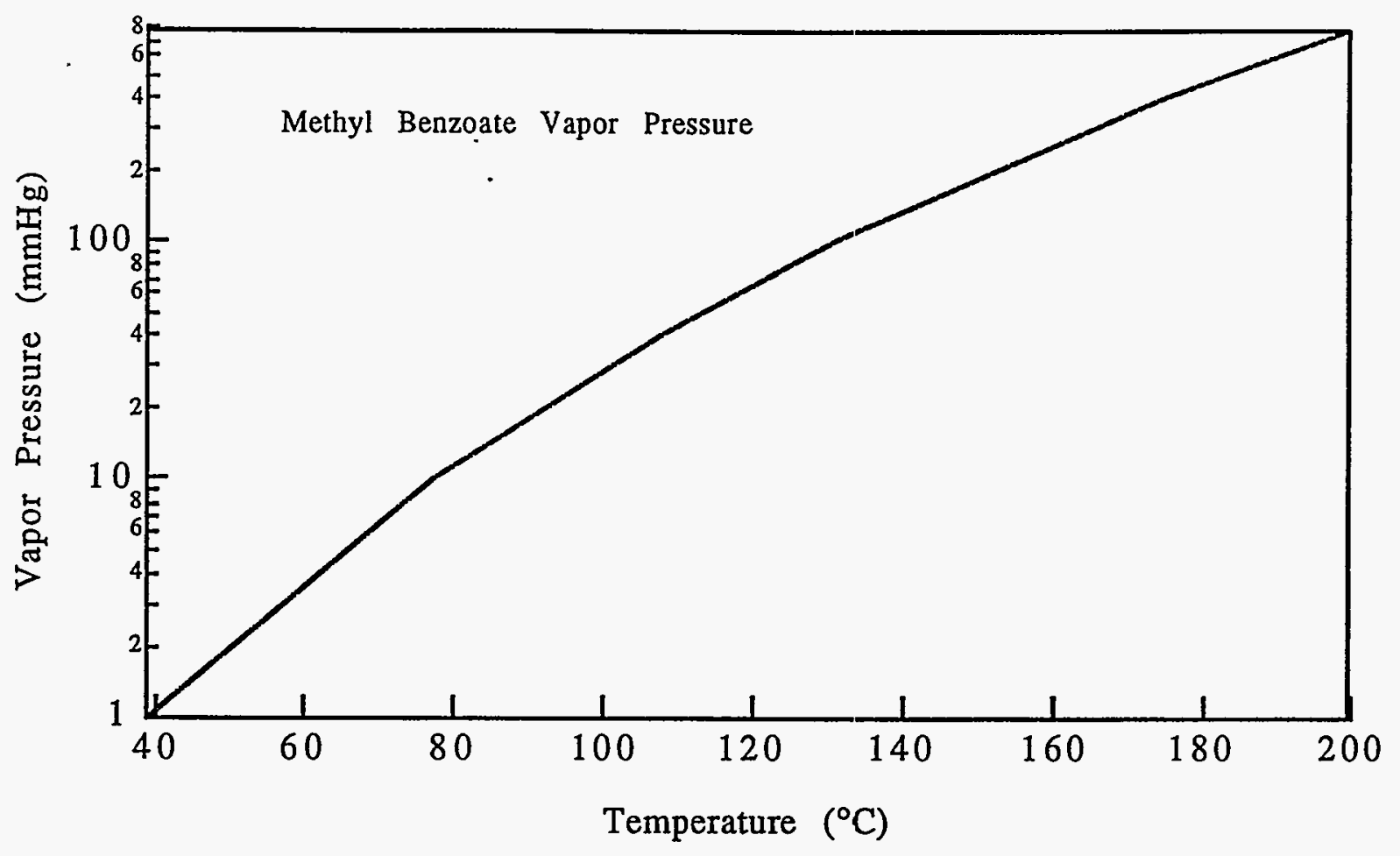


Technical Information Department - Lawrence Livermore National Laboratory University of California - Livermore, California 94551

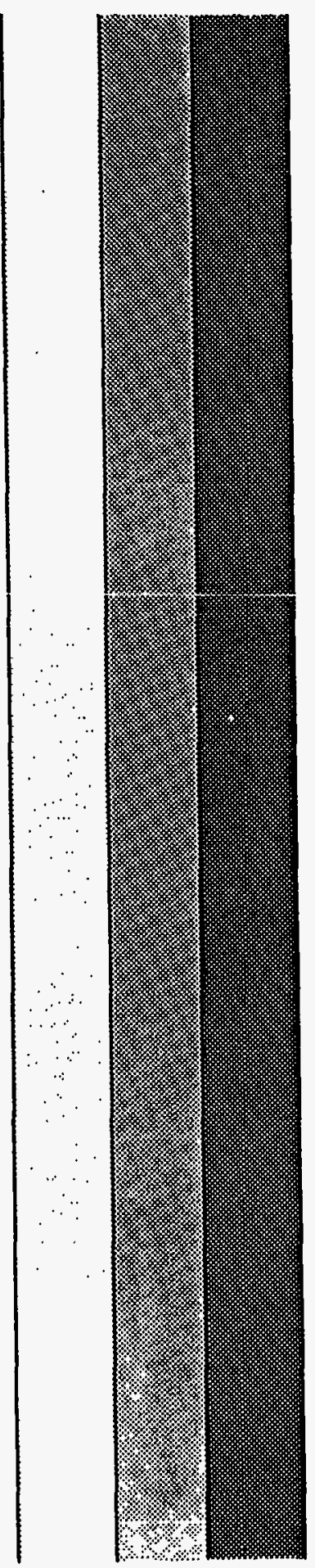

